\section{SAT0180 EVALUATION OF FRAILTY IN SJ GREN'S SYNDROME: CREATION OF A FRAILTY INDEX}

Raffaella Izzo ${ }^{1}$, Marco Canevelli ${ }^{2}$, Serena Colafrancesco ${ }^{1}$, Antonina Minniti ${ }^{1}$, Angelica Gattamelata ${ }^{1}$, Francesca Arienzo ${ }^{1}$, Federica Quarata ${ }^{2}$, Francesca Remiddi ${ }^{2}$, Valeria Raparelli ${ }^{3}$, Giuseppe Bruno ${ }^{2}$, Roberta Priori ${ }^{1}$, Guido Valesini'. ${ }^{1}$ Policlinico Umberto I, Rheumatology, Department of Internal Medicine and Medical Specialties, Sapienza University of Rome, Rome, Italy, Roma, Italy; ${ }^{2}$ Policlinico Umberto I, Neurology and Psychiatry, Department of Neuroscience, Sapienza University of Rome, Rome, Italy, Roma, Italy; ${ }^{3}$ Policlinico Umberto I, Department of Sperimental Medicine, Sapienza University of Rome, Rome, Italy, Roma, Italy

Background: Frailty is a condition characterized by the reduction of the individual's homeostatic reserves, leading to an increased vulnerability to stressors and an increased risk of unfavourable events. The aging of the population and the consequent need to implement new paradigms of care and assistance, have given this tool a growing interest in many medical disciplines ${ }^{1}$. In rheumatology, however, the interest is still limited ${ }^{2}$. The Frailty Index (FI), developed on an arithmetical model of deficit accumulation, is an accurate tool for assessing frailty, providing an estimate of the biological aging.

Objectives: Creation of a $\mathrm{Fl}$ to be used in clinical practice in patients with Sjögren's syndrome (SS) and evaluation of the correlations with patient's age, duration of illness, activity and disease damage at baseline and in the following 5 years.

Methods: The FI is composed by a checklist of non-predefined variables (deficits) constituted by symptoms, signs, diseases, disabilities, and laboratory findings. The deficits must meet these criteria: age-related; associated with negative outcomes; multidimensional (referring to different domains of the health status); present in at least $1 \%$, but not more than $80 \%$ of the sample. To each variable is assigned the value of 0 (= no deficit) or 1 (= deficit). The $\mathrm{FI}$ is the ratio between deficits presented by the individual and the total number of deficits considered, thus providing a measure of frailty ranging between 0 (no frailty) and 1 (maximum of frailty) ${ }^{3}$. A FI was developed for patients with SS consisting of 43 items (17 comorbidities, 14 signs and symptoms, 5 disabilities and 7 laboratory findings). Statistical analysis was performed with Spearman's test for correlation assessment, the Mann Whitney test for comparing non-parametric variables was used.

Results: FI was administered to a first small group of 30 female consecutive patients recruited as outpatients at the clinic dedicated to SS. The average age was $57.2 \mathrm{yrs}$, mean age at diagnosis $52.7 \mathrm{yrs}$ and average disease duration $4.7 \mathrm{yrs}$. At the time of completing the $\mathrm{Fl}$, the average disease activity (ESSDAl) was 3.4, the mean value of the damage (SjSDDI) 1.6 and the average score of $\mathrm{FI}$ equal to 0.21 . A statistically significant correlation between $\mathrm{FI}$ and age has been reported $(\mathrm{p}=$ 0.017). No significant correlations between frailty and duration, activity and disease damage have been highlighted at the moment.

Conclusion: For the first time a FI was developed for patients with SS consisting of 43 items. The data shows a relationship between age andFI. The correlation is statistically significant, similarly to what is reported in the literature for other conditions. This confirms that $\mathrm{Fl}$ is indeed an objective marker of aging and even though the sample population is young (average age $=57.2$ years), $\mathrm{Fl}$ maintains its main properties. This tool can be used to assess the health status of patients, making it possible to identify those at greater risk of trajectories or unfavourable outcomes. It is currently being administered the $\mathrm{FI}$ to patients with SS whose clinical course will be evaluated in the next 5 years (complications, mortality, hospitalization, institutionalization and disability).

\section{REFERENCES}

[1] Canevelli $\mathrm{M}$, et al, Promoting the Assessment of Frailty in the Clinical Approach to Cognitive Disorders, Front. Aging Neurosc 2017

[2] Rockwood MR, et al, FI to Measure Health Status in People with SSc, J Rheum 2014

[3] Searle SD, et al, A standard procedure for creating a FI, BMC Geriatrics 2008

Disclosure of Interests: None declared DOI: 10.1136/annrheumdis-2019-eular.7726

\section{SAT0181 \\ CONTRACEPTIVE COUNSELING AND USE AMONG WOMEN WITH SYSTEMIC LUPUS ERYTHEMATOSUS AT RISK FOR UNPLANNED PREGNANCY}

Francisca Aguiar ${ }^{1}$, Rui Costa ${ }^{2}$, Iva Brito ${ }^{1,3} .{ }^{1}$ Centro Hospitalar Universitário São João, Rheumatology, Porto, Portugal; ${ }^{2}$ Centro Hospitalar Universitário São João, Physical and Rehabilitiation Medicine, Porto, Portugal; ${ }^{3}$ Faculdade de Medicina da Universidade do Porto - FMUP, Porto, Portugal

Background: Systemic Lupus Erythematosus (SLE) is an autoimmune disease that primarily affects women of reproductive age. Disease activity and medication use can complicate pregnancies in SLE, due to the disease itself and/or exposure to teratogenic medications. Therefore, these patients should be counseled and are candidates for highly effective contraceptive methods.

Objectives: To examine contraceptive counseling and use among SLE patients attending our Rheumatology Department.

Methods: Cross-sectional study in which women aged 15-50 followed in our Rheumatology Centre with SLE diagnosis completed a researcheradministered survey. Premenopausal women who were sexually active were considered at risk of pregnancy. We compared self-reported rates of contraceptive counseling and use, stratified by treatment with teratogenic medications, and by history of thrombosis or antiphospholipid antibodies (aPL). The statistical analysis was performed using SPSS 23.0, and $p<0.05$ was taken to indicate statistical significance.

Results: 95 women were interviewed, of these, 60 were considered to be at risk for unplanned pregnancy. Their median age was 36 years (range 17-48), and median disease duration 9.9 years (range 0.25-37.0). 85\% were aware of the complications associated with pregnancy in their medical condition and $73.3 \%$ had received contraceptive counseling. Fifty-six patients (93.3\%) reported consistent contraceptive use. Younger patients were more likely to have received contraceptive counseling (35.0 [17-46] years versus 42.5 [20-48] years, $p=0.021$ ). Counseling was more frequently reported by patients with higher educational level $(p=0.026)$ Those who were counseled were using more effective contraceptives and in logistic regression contraceptive counseling was a predictor of highly effective contraception use ( $O R=13.1, p<0.0001)$.

Women using teratogenic medications or with a history of thrombosis were no more likely to have received contraceptive counseling or to use more effective contraceptives. Those with positive aPL were using more effective contraceptives $(p=0.024)$. In our model, having a high school degree and positive lupus anticoagulant predicted contraceptive counseling (OR=12.6, $\mathrm{p}=0.041 ; \mathrm{OR}=3.1, \mathrm{p}=0.02$, respectively).

Conclusion: This study highlights the importance of contraceptive counseling in SLE patients at risk for unplanned pregnancy. A multidisciplinary team including rheumatologists, gynecologists and family phsycians is needed to improve the education and provision of adequate contraceptive counseling to these women.

\section{REFERENCES}

[1] Bundhun PK, Soogund MZ, Huang F. Impact of systemic lupus erythematosus on maternal and fetal outcomes following pregnancy: a meta-analysis of studies published between years 2001-2016. J Autoimmun. 2017;79:17-27

[2] Yazdany J, et al. Contraceptive counseling and use among women with systemic lupus erythematosus: a gap in health care quality?.Arthritis Care Res (Hoboken). 2011;63:358-65.

Disclosure of Interests: None declared

DOI: 10.1136/annrheumdis-2019-eular.3624

\section{\begin{tabular}{|l|l}
\hline SAT0182 DYSLIPIDEMIA, HYPERTENSION, LUPUS NEPHRITIS \\
\hline
\end{tabular} AND HIGHER PREDNISONE USAGE CONTRIBUTE TO EVOLUTION OF CAROTID INTIMA-MEDIA THICKNESS IN MILD SYSTEMIC LUPUS ERYTHEMATOSUS: A 7-YEAR FOLLOW-UP STUDY}

Sofia Ajeganova $^{1,2}$, Thomas Gustafsson², Tomas Jogestrand ${ }^{2}$, Ingiäld Hafström², Johan Frostegård'. ${ }^{1}$ Vrije Universiteit Brussel, Brussels, Belgium; ${ }^{2}$ Karolinska Institutet, Stockholm, Sweden

Background: In SLE accelerated progression of carotid plaque and contribution of inflammatory factors for premature vascular changes have been reported. Effect of classical risk factors on progression of carotid intima- 
media thickness (cIMT) in patients with SLE in comparison with population controls is not clear.

Objectives: To examine in SLE and population controls (1)prevalence of risk factors overtime; (2)evolution of cIMT; (3)risk factors promoting cIMT evolution.

Methods: The study sample originated from the SLEVIC cohort (SLE vascular impact cohort study) which included consecutive patients with SLE and age and sex matched population controls. Seven years after inclusion all participants were asked to take part in the follow-up investigation. The standardized data collection and carotid ultrasound were performed at two assessments, 7 years apart. Effect of risk factors on clMT overtime was examined with linear mixed models adjusted for age, sex and traditional CV risk factors.

Results: A total of 77 patients with SLE (68\% of original cohort), $87 \%$ females, at inclusion mean age 47 years, disease duration 11.4 years, SLEDAI 3.0, SLICC/ACR 1.1, and 74 controls (61\% of original cohort), $89 \%$ females, mean age 51years, completed 7-years follow-up.

At inclusion, patients with SLE were younger and had lower levels of LDL than controls but were more likely to have hypertension and higher levels of triglycerides (TG). Between the assessments both groups were measured with an increase in blood pressure, levels of total cholesterol (TC), LDL, but at follow-up the patients still had lower TC, LDL and HDL levels and higher TG than controls. At both assessments, patients used more frequent antihypertensive and aspirin than controls, $p<0.001$.

The mean cIMT increased statistically significant in patients and controls, average absolute progression of $0.009 \mathrm{~mm} / \mathrm{year}$ in patients and 0.011 $\mathrm{mm} /$ year in controls, $\mathrm{p}<0.001$ for change in both groups, with no intergroup difference, $\mathrm{p}=0.867$ age- and sex-adjusted.

In multivariate analysis, the patients showed a statistically significant association between mean cIMT overtime and higher systolic blood pressure, lower levels of $\mathrm{HDL}$, higher TC/HDL- and LDL/HDL-ratio, higher triglycerides, dyslipidemia and detection with (any) carotid plaque at inclusion. In the control group, lower levels of $\mathrm{HDL}$, history of dyslipidemia and finding with bilateral plaque at inclusion were associated with cIMT overtime. At follow-up, hypertension and blood lipid measures in patients (with exception for TG) and HDL in controls were still significantly associated with cIMT overtime. Of all, the strongest association was shown for HDL in both patients and controls. Effect of other cardiometabolic risk factors at inclusion and cumulative risks were not significant after adjustment for age and sex.

In the patients, history of nephritis at inclusion and follow-up, and a higher average dose of prednisone used since diagnosis, but not other treatments, were associated with cIMT overtime.

Conclusion: We observed similar progression of cIMT over 7 years in patients with long-standing mild well-controlled SLE disease and population controls. Our findings suggest importance of recognition with dyslipidemia and hypertension, and support recommendation to limit use of corticosteroids in patients with SLE as a part of CV risk management. The factors protecting or promoting atherosclerotic progression should be further explored.

Disclosure of Interests: Sofia Ajeganova: None declared, Thomas Gustafsson: None declared, Tomas Jogestrand: None declared, Ingiäld Hafström: None declared, Johan Frostegård Shareholder of: Minor shareholder and inventor in startup-company Athera Biotechnologies, but they do not produce drugs yet and rheumatology is not in their focus.

DOI: 10.1136/annrheumdis-2019-eular.4863

\section{SAT0183 LUPUS NEPHRITIS: A RETROSPECTIVE LONGITUDINAL STUDY LOOKING FOR CHRONIC RENAL DISEASE ASSOCIATED FACTORS, FROM THREE SOUTH- EUROPEAN COHORTS OF PATIENTS IN FOLLOW-UP SINCE 2000}

Irene Altabás González¹, Jose M. Pego-Reigosa ${ }^{1}$, Jose Maria González², Francisco Rubiño ${ }^{2}$, Chiara Stagnaro ${ }^{3}$, Chiara Tani ${ }^{3}$, Carlos Rodriguez-Lozano ${ }^{2}$, Maria Celia Erausquin ${ }^{2}$, Juan Carlos Quevedo-Abeledo², Iñigo Hernández ${ }^{1}$, Marta Mosca ${ }^{3}$, Iñigo Rua-Figueroa ${ }^{2}{ }^{1}$ Hospital do Meixoeiro, Vigo, Spain; ${ }^{2}$ Hospital Doctor Negrín, Las Palmas de Gran Canaria, Spain; ${ }^{3}$ University of Pisa, Pisa, Italy

Background: Renal involvement in systemic lupus erythematosus (SLE) is the most frequent severe manifestation and carries a bad prognosis.

Objectives: To analyze the outcome of lupus nephritis (LN) in terms of chronic kidney disease development (CKD).

Methods: Design: multicentre restrospective observational study. Patients: SLE patients (ACR97) with biopsy proven LN attending to three South
European Rheumatology departments. Variables: demographics, SLE related variables, including global activity (SLEDAI-2K), renal flares, therapies, ACR response criteria and CKD. Statistical analysis: descriptive bivariate and multivariate analysis exploring factors associated to CKD.

Results: Seventy-six patients with biopsy-proven LN were included, 90,7\% female; mean age: 33 years; mean disease: duration 14 years; mean follow-up time (since LN diagnosis): 8,5 years. LN class III, IV and V were present in $22 \%, 75 \%$ and $3 \%$ of the cases, respectively. At LN diagnosis $68(89 \%)$ patients had a severe renal flare. Forty-one (56.1\%) and 49 $(64.4 \%)$ had HTA and nephrotic syndrome, respectively. The mean $24 \mathrm{~h}$ proteinuria levels at LN diagnosis was $4,6 \mathrm{~g}$. Mean SLEDAI-2K at the time of flare was 20.3 , with $69(65.7 \%)$ patients having an extrarenal flare.

The treatments used to induce remission were: glucocorticoids (100\%) pulses M-prednisolone in $49(64 \%)$ and oral prednisone (mean starting dose): $43 \mathrm{mg} /$ day $( \pm 20.6)$; intravenous cyclophosphamide in $42(55 \%)$ patients; mycophenolate mofetil (MMF) in 21 (27\%) patients; calcineurin inhibitors in $5(11 \%)$ patients; rituximab in $4(5.2 \%)$ patients; and oral cyclophosphamide in $4(5,2 \%)$ patients. Forty-eight $(63 \%)$ patients were receiving hydroxychloroquine. MMF was the immunosuppressant (IS) more frequently used (52\%) as maintenance therapy.

At 3,6 and 12th months, the mean proteinuria was $2.3 \mathrm{~g} / 24 \mathrm{~h}, 1.53 \mathrm{~g} / 24 \mathrm{~h}$, $1.1 \mathrm{~g} / 24 \mathrm{~h}$, respectively $(\mathrm{p}<0.001)$. Fifty-five $(77,5 \%)$ of patients achieved complete response and $61(84.7 \%)$ presented complete or partial response. Median time to renal remission: 12.5 months $(6,17.5)$. In 32 patients $(42 \%)$ it was possible to discontinue IS. Sixteen $(21.9 \%)$ patients developed CKD, 4 (5.3\%) needing dialysis and $1(0.76 \%)$ renal transplantation. Serious infection was recorded in $23(34.8 \%)$ patients. Five $(6.6 \%)$ patients died ( 2 cardiovascular cause).

In the bivariate study, the following variables were significantly associated with CKD: male sex, hypertension, ACEI drugs, severe infection after LN temporal dyalisis, non ACR renal response, non use of hydroxychloroquine, time to achieve $10 \mathrm{mg} /$ day of prednisone, previous creatinine to $\mathrm{LN}$, maximum creatinine at LN, hyperlipidemia at 3 months of $L N$, active urinary sediment at 12 months, creatinine at 6 and 12 months, proteinuria at 6 and 12 months.

In the logistic regression model, using genetic algorithms, we found that proteinuria at 6 months was significantly associated with CKD (OR:2.95; $95 \% \mathrm{Cl} 1.19,9.29, \mathrm{p}=0.03)$. Hypertension and male sex were marginally associated ( $p=0.06$, both).

Conclusion: A considerable percentage of $\mathrm{LN}$ patients developed renal chronic failure $(21,9 \%)$. A high percentage of ACR response was achieved using medium dose $(40 \mathrm{mg})$ of glucocorticoids for induction. A significative reduction of proteinuria was achieved at 3 months, but proteinuria at 6 months was the only factor finally associated with CKD.

Disclosure of Interests: Irene Altabás González: None declared, Jose M Pego-Reigosa: None declared, Jose Maria González: None declared, Francisco Rubiño: None declared, Chiara Stagnaro: None declared, Chiara Tani: None declared, Carlos Rodriguez-Lozano: None declared, Maria Celia Erausquin: None declared, Juan Carlos Quevedo-Abeledo: None declared, Iñigo Hernández: None declared, Marta Mosca Paid instructor for: GlaxoSmithKline, Lilly, UCB, Iñigo Rua-Figueroa: None declared DOI: 10.1136/annrheumdis-2019-eular.5497

\section{SAT0184 ASSOCIATION OF SERUM AND URINE LEVELS OF TWEAK, MCP-1 AND NGAL WITH DISEASE ACTIVITY IN SYSTEMIC LUPUS ERYTHEMATOSUS}

Safak Mirioglu ${ }^{1}$, Suzan Çinar ${ }^{2}$, Halil Yazici ${ }^{3}$, Ahmet Gül ${ }^{4}$, Lale Ocal ${ }^{4}$, Murat Inanc ${ }^{4}$, Bahar Artim-Esen ${ }^{4} .{ }^{1}$ Istanbul University, Istanbul Faculty of Medicine, Department of Internal Medicine, Istanbul, Turkey, ${ }^{2}$ Istanbul University, Aziz Sancar Institute of Experimental Medicine, Department of Immunology, Istanbul, Turkey, ${ }^{3}$ Istanbul University, Istanbul Faculty of Medicine, Department of Internal Medicine, Division of Nephrology, Istanbul, Turkey, ${ }^{4}$ Istanbul University, Istanbul Faculty of Medicine, Department of Internal Medicine, Division of Rheumatology, Istanbul, Turkey

Background: TWEAK, MCP-1 and NGAL, mediators in pathogenesis of systemic lupus erythematosus (SLE), are proinflammatory cytokines/chemokines that are thought as potential biomarkers reflecting disease activity (1).

Objectives: In this study, we aimed to investigate the association of serum (s) and urine (u) levels of TWEAK, MCP-1 and NGAL with disease activity in both renal and non-renal SLE.

Methods: Thirty active patients with SLE (15 renal and 15 non-renal) were recruited. Thirty-one inactive patients with SLE (16 renal and 15 